International Journal of Recent advances in Physics (IJRAP) Vol.4, No.3, August 2015

\title{
BOUND- STATE SOLUTION OF SCHRODINGER EQUATION WITH HULTHEN PLUS GENERALIZED EXPONENTIAL COULOMB POTENTIAL USING NIKIFOROV-UVAROV METHOD
}

\author{
Ituen. B. Okon ${ }^{1}$ and Oyebola Popoola ${ }^{2}$ \\ ${ }^{1}$ Department of Physics, University of Uyo, Nigeria. \\ ${ }^{2}$ Department of Physics, University of Ibadan, Nigeria.
}

\begin{abstract}
We apply an approximation to centrifugal term to find bound state solutions to Schrodinger equation with Hulthen Plus generalized exponential Coulomb potential Using Nikiforov-Uvarov Method. Using this method, we obtained the energy-eigen value and the total wave function. We implement $C++$ algorithm, to obtained the numerical values of the energy for different quantum state starting from the first excited state for different values of the screening parameter.
\end{abstract}

\section{Keywords}

Nikiforov-Uvarov method, Schrodinger equation, Hulthen plus exponential Coulomb potential. PACS: 03.65.Ge, 03.65. $-\mathrm{w}$

\section{Introduction}

The exact and approximate bound state solution to Schrodinger wave equation has arouse the interest of many authors for decades. Theses solutions are very important with enormous applications in physical sciences and quantum field theory [1-9]. Finding the solution of the wave equations whether relativistic or non relativistic help in quantum mechanical studies. Different authors have used different techniques and methods for this purpose among them are: Analytical Nikiforov-Uvarov method [10-12], factorization method [13], super-symmetric quantum approach [14-18], shape invariant method [19], the ansatz solution approach [20-22], exact quantization rule [23-24], tridiagonalisation program[25], variational method [26] and many others.

However, these techniques are applicable for selected potentials, few amongst them are : RosenMorse, Hulthen, pseudoharmonic, Poschl-Teller, kratzer fues and Mie-Type potentials[27-30]. The study of the proposed potential is very essential in investigating the interaction existing between diatomic molecules. 
The main aim of this paper is to present and study Hulthen plus generalized exponential coulomb potential from which other potentials are deduced as special cases. This article is organized as follows: section 1 is the introduction, section 2 gives a brief discussion of Nikiforov-Uvarov method. The method used in this article reproduces accurate analytical solutions for many differential equations with significant application in physics, for example it can be used for equation of Hermite, Laguerre and Jacobi. Section 3 gives the radial solution to Schrodinger equation with the proposed potential, section 4 gives the result and discussion while section 5 gives the numerical computation of the resulting energy equation.

The proposed potential is given by

$$
V(r)=\frac{-V_{0} e^{-2 \alpha r}}{\left(1-e^{-2 \alpha r}\right)}-\frac{A e^{-\alpha r}}{r}
$$

Hulthen potential describes the interaction existing between diatomic molecules but addition of the second term which is the exponential coulomb potential ascertain the attractive force that holds the molecules together. However, this force exist as Coulomb force.

\section{Review of Nikiforov-Uvarov method.}

The NU method is based on reducing second order linear differential equation to a generalized equation of hyper-geometric type .This method provides solution in terms of special orthogonal functions as well as corresponding energy eigen value. With appropriate coordinate transformation, $s=s(x)$, this equation can be written as

$$
\psi^{\prime \prime}(s)+\frac{\tilde{\tau}(s)}{\sigma(s)} \psi^{\prime}(s)+\frac{\tilde{\sigma}(s)}{\sigma^{2}(s)} \psi(s)=0
$$

In order to find the exact solution to equation (2), we set the wave function as

$$
\psi(s)=\phi(s) \chi(s)
$$

Substituting equation (3) into equation (2) gives the hyper-geometric equation

$$
\sigma(s) \chi^{\prime \prime}(s)+\tau(s) \chi^{\prime}(s)+\lambda \chi(s)=0
$$

Where the wave function is defined as the logarithmic derivative

$$
\frac{\phi^{\prime}(s)}{\phi(s)}=\frac{\pi(s)}{\sigma(s)}
$$

Where $\pi(s)$ is at most polynomial of first degree. However, the hyper-geometric type function $\chi(s)$ in equation (4) for a fixed $\mathrm{n}$ can be express in Rodrigue relation 


$$
\chi_{n}(s)=\frac{B_{n}(s)}{\rho(s)} \frac{d^{n}}{d s^{n}}\left[\sigma^{n}(s) \rho(s)\right]
$$

$B_{n}$ is the normalization constant and the weight function $\rho(s)$ satisfies the condition

$$
\frac{d}{d s}(\sigma(s) \rho(s))=\tau(s) \rho(s)
$$

Also $\quad \tau(s)=\tilde{\tau}(s)+2 \pi(s)$

In order to accomplish the conditions imposed on the weight function $\rho(s)$, it is necessary that the classical orthogonal polynomial $\tau(s)$ be equal to zero, so that its derivative be less than zero. That is

$$
\frac{d \tau(s)}{d s}<0
$$

Therefore the function $\pi(s)$ and the parameter $\lambda$ required for the NU method are defined as follows:

$$
\begin{gathered}
\pi(s)=\frac{\sigma^{\prime}-\tilde{\tau}}{2} \pm \sqrt{\left(\frac{\sigma^{\prime}-\tilde{\tau}}{2}\right)^{2}-\tilde{\sigma}+k \sigma} \\
\lambda=k+\pi^{\prime}(s)
\end{gathered}
$$

$k$ values in equation (10) are possible to evaluate if the expression under the square root must be square of polynomials. This is possible if and only if its descriminant is zero. With this a new eigen equation becomes

$$
\lambda_{n}=\frac{n d \tau(s)}{d s}-\frac{n(n-1)}{2} \frac{d^{2} \sigma}{d s^{2}}
$$

$$
\mathrm{n}=0,1,2-
$$

Where $\tau(s)$ is defined in equation (8) and on comparing equation (11) to (12), we can now obtained the energy eigen value.

\section{Solution of Schrodinger Equation}

The Schrodinger wave equation is given by

$$
\frac{d^{2} \psi(r)}{d r^{2}}+\frac{2 \mu}{\hbar^{2}}\left[E-V(r)-\frac{\hbar^{2} l(l+1)}{2 \mu r^{2}}\right] \psi(r)=0
$$

Substituting equation (1) into (13) gives 
International Journal of Recent advances in Physics (IJRAP) Vol.4, No.3, August 2015

$\frac{d^{2} \psi(r)}{d r^{2}}+\frac{2 \mu}{\hbar^{2}}\left[E+\frac{V_{0} e^{-2 \alpha r}}{\left(1-e^{-2 \alpha r}\right)}+\frac{A e^{-\alpha r}}{r}-\frac{\hbar^{2} l(l+1)}{2 \mu r^{2}}\right] \psi(r)=0$

Lets define some approximation scheme

$\frac{1}{r^{2}}=\frac{4 \alpha^{2} e^{-2 \alpha r}}{\left(1-e^{-2 \alpha r}\right)^{2}}$ and $\frac{1}{r}=\frac{2 \alpha e^{-\alpha r}}{\left(1-e^{-2 \alpha r}\right)}$

Substituting equation (15) into equation (14) with the transformation $s=e^{-2 \alpha r}$ gives

$\frac{d^{2} \psi(s)}{d s^{2}}+\frac{(1-s)}{s(1-s)} \frac{d \psi(s)}{d s}+\frac{1}{s^{2}(1-s)^{2}}\left[\left(-\mathcal{E}^{2}-\delta^{2}-\sigma_{1}\right) s^{2}+\left(2 \varepsilon^{2}+\delta^{2}+\sigma_{1}-l(l+1)\right) s-\varepsilon^{2}\right] \psi(s)=0$

Where

$$
\left[\varepsilon^{2}=-\frac{2 \mu E}{4 \hbar^{2} \alpha^{2}}, \delta^{2}=\frac{2 \mu V_{0}}{4 \hbar^{2} \alpha^{2}}, \sigma_{1}=\frac{\mu A}{\hbar^{2} \alpha}\right]
$$

Comparing equation (16) to equation (2) we obtained the followings:

$$
\tilde{\tau}(s)=(1-s), \sigma(s)=s(1-s), \tilde{\sigma}(s)=-\left(\varepsilon^{2}+\delta^{2}+\sigma_{1}\right) s^{2}+\left(2 \varepsilon^{2}+\delta^{2}+\sigma_{1}-l(l+1)\right) s-\varepsilon^{2}
$$

Then, using equation (10), the polynomial equation $\pi(s)$ then become

$$
\pi(s)=\frac{-s}{2} \pm \sqrt{\left(\frac{1}{4}+\varepsilon^{2}+\delta^{2}+\sigma_{1}-k\right) s^{2}-\left(2 \varepsilon^{2}+\delta^{2}+\sigma_{1}-l(l+1)-k\right) s+\varepsilon^{2}}
$$

To find the value of $k$ we consider the discriminant such that $b^{2}-4 a c=0$

Hence,

$$
\begin{aligned}
& k_{1}=-\left(l(l+1)-\sigma_{1}-\delta^{2}\right)+\varepsilon \sqrt{4 l(l+1)+1} \\
& k_{2}=-\left(l(l+1)-\sigma_{1}-\delta^{2}\right)-\varepsilon \sqrt{4 l(l+1)+1}
\end{aligned}
$$

Substituting the values of $k_{1}$ and $k_{2}$ into equation (19), then the four values of $\pi(s)$ is given below. 
International Journal of Recent advances in Physics (IJRAP) Vol.4, No.3, August 2015

$$
\pi(s)=\frac{-s}{2} \pm\left[\begin{array}{ccc}
{\left[\left(\frac{1}{2} \sqrt{(4 l(l+1)+1}+\varepsilon\right) s-\varepsilon\right],} & k_{1}= & -\left(l(l+1)-\sigma_{1}-\delta^{2}\right)+\varepsilon \sqrt{4 l(l+1)+1} \\
{\left[\left(\frac{1}{2} \sqrt{(4 l(l+1)+1)}+\varepsilon\right) s+\varepsilon\right],} & k_{2}= & -\left(l(l+1)-\sigma_{1}-\delta^{2}\right)-\varepsilon \sqrt{4 l(l+1)+1}
\end{array}\right]
$$

$\pi(s)$ has four solutions and one of these solutions satisfied bound state condition which is

$$
\pi(s)=-\frac{s}{2}-\left[\left(\frac{1}{2} \sqrt{4 l(l+1)+1}+\varepsilon\right) s-\varepsilon\right]
$$

Using equation $(8) \quad \tau(s)=1-2 s-(\sqrt{4 l(l+1)+1}) s-2 \varepsilon s+2 \varepsilon$

$$
\text { Which implies that } \quad \tau^{\prime}(s)=[-2-(\sqrt{4 l(l+1)+1})-2 \varepsilon]<0
$$

Equation (23) satisfies bound state condition. The energy eigen value can be calculated using equation (11)

$$
\lambda=-l(l+1)+\delta_{1}+\delta^{2}-\varepsilon \sqrt{4 l(l+1)+1}-\frac{1}{2}-\frac{1}{2} \sqrt{4 l(l+1)+1}-\varepsilon
$$

\section{Applying equation (12)}

$$
\lambda_{n}=-2 n-n \sqrt{\left(4 \sigma_{2}+4 l(l+1)+1\right)}-2 n \sqrt{\left(\varepsilon^{2}+\sigma_{3}\right)}
$$

Equating equation (24) and (25) gives the energy equation as follow

$$
\varepsilon=\left[\frac{\frac{1}{2}-2 n+\sqrt{4 l(l+1)+1}\left(\frac{1}{2}-n\right)+l(l+1)-\sigma_{1}-\delta^{2}}{(2 n+\sqrt{4 l(l+1)+1}-1)}\right]
$$

Substituting parameters in equation (17) into equation (26) gives 


$$
E_{n l}=-\frac{2 \hbar^{2} \alpha^{2}}{\mu}\left[\frac{\frac{1}{2}-2 n+\sqrt{4 l(l+1)+1}\left(\frac{1}{2}-n\right)+l(l+1)-\frac{\mu A}{\alpha \hbar^{2}}-\frac{2 \mu v_{0}}{4 \hbar^{2} \alpha^{2}}}{(2 n+\sqrt{4 l(l+1)+1}-1)}\right]^{2}
$$

Equation (27) is the energy of the proposed potential.

\section{Calculation of the wave function}

By using equation (5)

$$
\frac{\phi^{1}(s)}{\phi(s)}=\frac{\pi(s)}{\sigma(s)} \Rightarrow \frac{\phi^{1}(s)}{\phi(s)}=-\frac{1}{2(1-s)}-\frac{1}{2 s(1-s)} \sqrt{4 l(l+1)+1}+\frac{\varepsilon}{(1-s)}-\frac{\varepsilon}{s(1-s)}
$$

Taking integral of equation (28) and engaging mathematical algebraic simplification gives

$$
\phi(s)=[1-s]^{\left(\frac{1}{2}+\frac{1}{2} \sqrt{4 l(l+1)+1}\right)} s^{-\mathcal{E}}
$$

Equation (29) is the solution to the first part of the wave equation. To determine the second solution of the wave function, it is necessary to compute the weight function .

\section{Calculation of weight function}

Using equation (7)

$$
\frac{d}{d s}(\sigma(s) \rho(s))=\tau(s) \rho(s) \Rightarrow \int \frac{\rho^{\prime}(s)}{\rho(s)} d s=\int \frac{\tau(s)-\sigma^{\prime}(s)}{\sigma(s)} d s
$$

Then the weight function can be given as

$$
\rho(s)=[1-s]^{(\sqrt{4 l(l+1)+1})} s^{2 \varepsilon}
$$

Rewriting equation(31) in its Rodrigue form by making use of equation(6) gives

$$
Y_{n}(s)=B_{n}(s) s^{-2 \varepsilon}[1-s]^{-(\sqrt{4 l(l+1)+1})} \frac{d^{n}}{d s^{n}}\left[s^{n+2 \varepsilon}[1-s]^{n+(\sqrt{4 l(l+1)+1})}\right]
$$

Lets define standard associated Laguerre polynomial as

$$
\chi_{n}(s)=B_{n}(s) s^{-2 v}(1-s)^{-\mu} \frac{d^{n}}{d s^{n}}\left[s^{n+2 v}(1-s)^{n+\mu}\right]=B_{n}(s) P_{n}^{[\mu+2 v, \mu]}(1-s)
$$


Then re-writing equation (31) in terms of equation (32) gives the second part of the wave function as

$$
Y_{n}(s)=B_{n}(s) P_{n}^{[(\sqrt{4 l(l+1)+1}+2 \varepsilon) \cdot(\sqrt{4 l(l+1)+1})]}(1-s)
$$

Hence the total wave function is given by

$$
\psi(s)=\phi(s) Y_{n}(s)=B_{n}(s)[1-s]^{(\sqrt{4 l(l+1)+1})} s^{2 \varepsilon} P_{n}^{[(\sqrt{4 l(l+1)+1}+2 \varepsilon),(\sqrt{4 l(l+1)+1})]}(1-s)
$$

\section{Result and Discussion}

By setting some potential parameters into equation (1), we obtain some well known potentials.

\subsection{Hulthen potential}

If $A=0$ in equation (1) then the potential reduces to Hulthen potential

$$
V(r)=\frac{-V_{0} e^{-2 \alpha r}}{\left(1-e^{-2 \alpha r}\right)}
$$

and the resulting energy of this potential is given by

$$
E_{n l}=-\frac{2 \hbar^{2} \alpha^{2}}{\mu}\left[\frac{\frac{1}{2}-2 n+\sqrt{4 l(l+1)+1}\left(\frac{1}{2}-n\right)+l(l+1)-\frac{2 \mu v_{0}}{4 \hbar^{2} \alpha^{2}}}{(2 n+\sqrt{4 l(l+1)+1}-1)}\right]^{2}
$$

\subsection{Generalized exponential coulomb potential}

If $v_{0}=0$ in equation (1), then, the potential reduces to generalized exponential potential

$$
-\frac{A e^{-\alpha r}}{r}
$$

The resulting energy equation is given by

$$
E_{n l}=-\frac{2 \hbar^{2} \alpha^{2}}{\mu}\left[\frac{\frac{1}{2}-2 n+\sqrt{4 l(l+1)+1}\left(\frac{1}{2}-n\right)+l(l+1)-\frac{\mu A}{\alpha \hbar^{2}}}{(2 n+\sqrt{4 l(l+1)+1}-1)}\right]^{2}
$$

5.0 Numerical result for Hulthen plus exponential coulomb potential for various value of $\alpha$ 
International Journal of Recent advances in Physics (IJRAP) Vol.4, No.3, August 2015

\section{Table1}

\begin{tabular}{|l|l|l|l|l|l|l|l|l|l|l|l|}
\hline$\eta$ & & $E_{n l}(\alpha=0.2)$ & $n$ & $l$ & $E_{n l}(\alpha=0.2)$ & $n$ & $l$ & $E_{n l}(\alpha=0.2)$ & $n$ & $l$ & $E_{n l}(\alpha=0.2)$ \\
\hline 1 & 0 & -0.0099999990 & 1 & 1 & -0.0625000000 & 1 & 2 & -0.1248444600 & 1 & 3 & -0.1980249900 \\
\hline 2 & 0 & -0.0001000000 & 2 & 1 & -0.0373777820 & 2 & 2 & -0.1056249900 & 2 & 3 & -0.1900960400 \\
\hline 3 & 0 & -0.0004000000 & 3 & 1 & -0.0272249970 & 3 & 2 & -0.0948639960 & 3 & 3 & -0.1849000000 \\
\hline 4 & 0 & -0.0012250000 & 4 & 1 & -0.0219040010 & 4 & 2 & -0.0880111200 & 4 & 3 & -0.1812326800 \\
\hline 5 & 0 & -0.0019360000 & 5 & 1 & -0.0186777750 & 5 & 2 & -0.0832734800 & 5 & 3 & -0.1785062100 \\
\hline 6 & 0 & -0.0024999997 & 6 & 1 & -0.0165306140 & 6 & 2 & -0.0798062460 & 6 & 3 & -0.1763999900 \\
\hline 7 & 0 & -0.0029469390 & 7 & 1 & -0.0150062490 & 7 & 2 & -0.0771604850 & 7 & 3 & -0.1747239800 \\
\hline 8 & 0 & -0.0033062496 & 8 & 1 & -0.0138716055 & 8 & 2 & -0.0750760140 & 8 & 3 & -0.1733586600 \\
\hline
\end{tabular}

Table 2

\begin{tabular}{|l|l|l|l|l|l|l|l|l|l|l|l|}
\hline & & $E_{n l}(\alpha=0.4)$ & $n$ & $l$ & $E_{n l}(\alpha=0.4)$ & $n$ & $l$ & $E_{n l}(\alpha=0.4)$ & $n$ & $l$ & $E_{n l}(\alpha=0.4)$ \\
\hline 1 & 0 & -0.51839995 & 1 & 1 & -12.6735990 & 1 & 2 & -28.7296000 & 1 & 3 & -47.6100000 \\
\hline 2 & 0 & -0.07840000 & 2 & 1 & -7.84000100 & 2 & 2 & -24.8003960 & 2 & 3 & -46.2400100 \\
\hline 3 & 0 & -0.37617780 & 3 & 1 & -5.85640050 & 3 & 2 & -22.5815070 & 3 & 3 & -45.3377760 \\
\hline 4 & 0 & -0.60839990 & 4 & 1 & -4.80486500 & 4 & 2 & -21.1600040 & 4 & 3 & -44.6987800 \\
\hline 5 & 0 & -0.77440010 & 5 & 1 & -4.16159960 & 5 & 2 & -20.1729340 & 5 & 3 & -44.2224960 \\
\hline 6 & 0 & -0.89617790 & 6 & 1 & -3.73041680 & 6 & 2 & -19.4481000 & 6 & 3 & -43.8538250 \\
\hline 7 & 0 & -0.98860407 & 7 & 1 & -3.42249970 & 7 & 2 & -18.8935090 & 7 & 3 & -43.5600050 \\
\hline 8 & 0 & -1.06090000 & 8 & 1 & -3.19217800 & 8 & 2 & -18.4556160 & 8 & 3 & -43.3203280 \\
\hline
\end{tabular}

Table 3

\begin{tabular}{|c|c|c|c|c|c|c|c|c|c|c|c|}
\hline & $l$ & $E_{n l}(\alpha=0.6)$ & $n$ & $l$ & $E_{n l}(\alpha=0.6)$ & $n$ & $l$ & $E_{n l}(\alpha=0.6)$ & & $l$ & $E_{n l}(\alpha=0.6)$ \\
\hline 1 & 0 & -5.47560070 & 1 & 1 & -301.717000 & 1 & 2 & -712.890140 & 1 & 3 & -1197.50600 \\
\hline 2 & 0 & -4.28490070 & 2 & 1 & -188.787640 & 2 & 2 & -619.263300 & 2 & 3 & -1167.17920 \\
\hline 3 & 0 & -12.5316030 & 3 & 1 & -142.205630 & 3 & 2 & -566.249760 & 3 & 3 & -1147.17710 \\
\hline 4 & 0 & -18.2756250 & 4 & 1 & -117.418930 & 4 & 2 & -532.225000 & 4 & 3 & -1132.99610 \\
\hline 5 & 0 & -22.2406620 & 5 & 1 & -102.212135 & 5 & 2 & -508.567080 & 5 & 3 & -1122.41770 \\
\hline 6 & 0 & -25.1001070 & 6 & 1 & -91.9955440 & 6 & 2 & -491.176500 & 6 & 3 & -1114.22470 \\
\hline 7 & 0 & -27.2484070 & 7 & 1 & -84.6860100 & 7 & 2 & -477.859620 & 7 & 3 & -1107.69190 \\
\hline 8 & 0 & -28.9175070 & 8 & 1 & -79.2100100 & 8 & 2 & -467.338100 & 8 & 3 & -1102.36080 \\
\hline
\end{tabular}


International Journal of Recent advances in Physics (IJRAP) Vol.4, No.3, August 2015

\section{Table 4}

\begin{tabular}{|c|c|c|c|c|c|c|c|c|c|c|c|}
\hline $\mathrm{n}$ & $l$ & $E_{n l}(\alpha=0.8)$ & $n$ & $l$ & $E_{n l}(\alpha=0.8)$ & $n$ & $l$ & $E_{n l}(\alpha=0.8)$ & & $l$ & $E_{n l}(\alpha=0.8)$ \\
\hline 1 & 0 & -29.5936010 & 1 & 1 & -2907.36620 & 1 & 2 & -7011.27050 & \begin{tabular}{l|l}
1 \\
\end{tabular} & 3 & -11854.85400 \\
\hline 2 & 0 & -56.5504000 & 2 & 1 & -1829.55790 & 2 & 2 & -6108.98500 & 2 & 3 & -11574.31800 \\
\hline 3 & 0 & -140.185610 & 3 & 1 & -1383.83970 & 3 & 2 & -5597.43400 & 3 & 3 & -11389.15800 \\
\hline 4 & 0 & -195.999970 & 4 & 1 & -1146.22860 & 4 & 2 & -5268.82470 & 4 & 3 & -11257.81600 \\
\hline 5 & 0 & -233.967640 & 5 & 1 & -1000.24610 & 5 & 2 & -5040.18850 & 5 & 3 & -11159.81000 \\
\hline \begin{tabular}{l|l}
6 \\
\end{tabular} & 0 & -261.145600 & 6 & 1 & -902.058350 & 6 & 2 & -4872.03960 & \begin{tabular}{l|l}
6 \\
\end{tabular} & 3 & -11083.88000 \\
\hline 7 & 0 & -281.472530 & 7 & 1 & -831.745500 & 7 & 2 & -4743.22950 & 7 & 3 & -11023.32100 \\
\hline 8 & 0 & -297.217600 & 8 & 1 & -779.030150 & 8 & 2 & -4641.42530 & 8 & 3 & -10973.89550 \\
\hline
\end{tabular}

Table5

\begin{tabular}{|l|l|l|l|l|l|l|l|l|l|l|l|}
\hline $\mathrm{n}$ & & $E_{n l}(\alpha=1.0)$ & $n$ & $l$ & $E_{n l}(\alpha=1.0)$ & $n$ & $l$ & $E_{n l}(\alpha=1.0)$ & $n$ & $l$ & $E_{n l}(\alpha=1.0)$ \\
\hline 1 & 0 & -110.25000 & 1 & 1 & -16965.059 & 1 & 2 & -41412.250 & 1 & 3 & -70291.250 \\
\hline 2 & 0 & -390.06244 & 2 & 1 & -10712.250 & 2 & 2 & -36147.510 & 2 & 3 & -68696.400 \\
\hline 3 & 0 & -890.02770 & 3 & 1 & -8122.5140 & 3 & 2 & -33160.406 & 3 & 3 & -67643.330 \\
\hline 4 & 0 & -1216.2654 & 4 & 1 & -6740.4097 & 4 & 2 & -31240.557 & 4 & 3 & -66896.125 \\
\hline 5 & 0 & -1436.4098 & 5 & 1 & -5890.5625 & 5 & 2 & -29904.287 & 5 & 3 & -66338.420 \\
\hline 6 & 0 & -1593.3401 & 6 & 1 & -5318.5767 & 6 & 2 & -28921.248 & 6 & 3 & -65906.290 \\
\hline 7 & 0 & -1710.4133 & 7 & 1 & -4908.7530 & 7 & 2 & -28168.025 & 7 & 3 & -65561.590 \\
\hline 8 & 0 & -1800.9410 & 8 & 1 & -4601.3604 & 8 & 2 & -27572.598 & 8 & 3 & -65280.242 \\
\hline
\end{tabular}

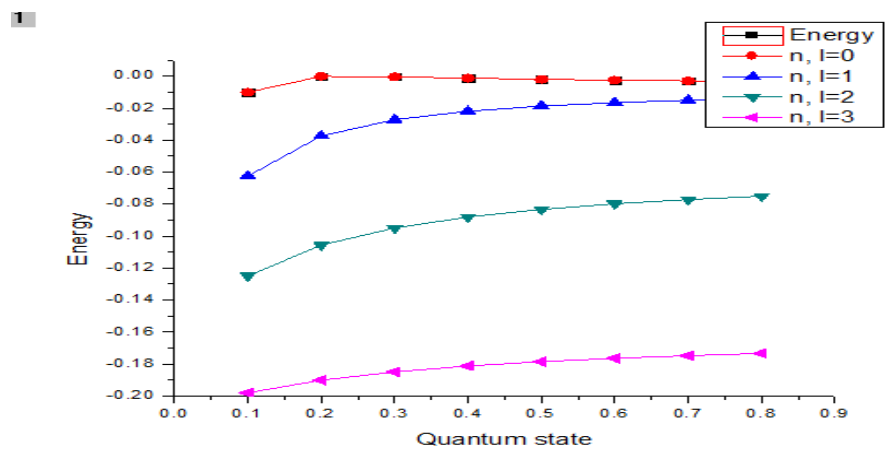

Figure 1. A graph of Hulthen plus modified exponential Coulomb potential for $\alpha=0.2$ 
International Journal of Recent advances in Physics (IJRAP) Vol.4, No.3, August 2015

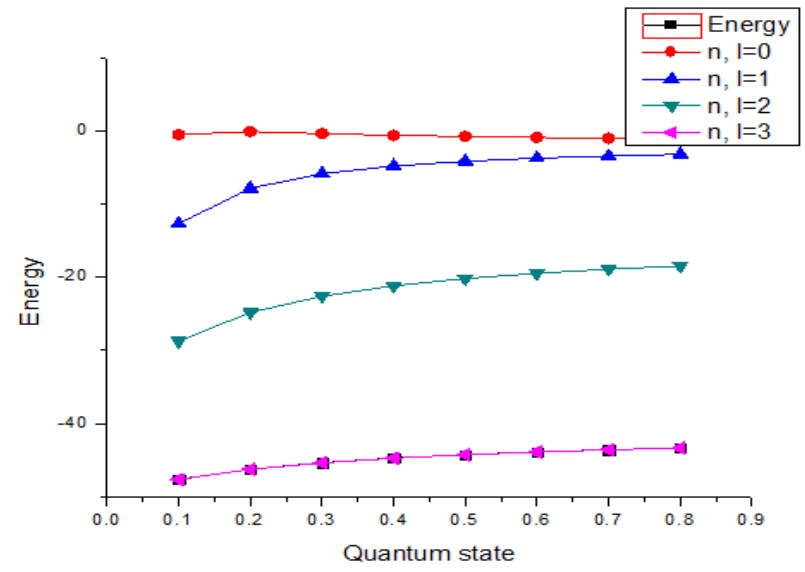

Figure 2: A graph of Hulthen plus modified exponential Coulomb potential for $\alpha=0.4$

\section{CONCLUSION}

In this paper, we have studied the bound state solution to Schrodinger equation using Hulthen plus generalized exponential coulomb potential via conventional Nikiforov-Uvarov method. We also obtained the energy of this potential and the total wave function in addition to energies of special cases. The Numerical result shows that the energy of this potential decreases with an increase in quantum state as shown in tables (1-5). However, the numerical result also shows that these energies apart from decreasing with an increase in screening parameter $\alpha$, have negative values to ascertain the bound state of the solution. Graphical illustration s shows a unique stability at different quantum state of the potential that describe the interaction existing in diatomic molecules.

These energies exist at different discrete energy eigen state illustrating the reason for stability of diatomic molecules.

\section{ACKNOWLEDGEMENT}

The authors are grateful to the anonymous reviewer for his useful comments which has significantly help for the improvement of this article.

\section{References}

[1] Okon I.B.,Ituen E.E., Popoola O and Antia A.D.( 2013), "Analytical Solutions of Schrodinger Equation with Mie-Type Potential Using Factorisation Method", International Journal of Recent Advances In Physics, Vol.2, No.2, pp1-7.

[2] Ikhdair S. M. and Sever R.( 2007). "A perturbative treatment for the bound states of the Hellmann potential", Journal of Molecular Structure, vol. 809, no. 1-3, pp. 103-113.

[3] Fl"ugge S., Practical Quantum Mechanics, Springer- Verlag, Berlin (1994).

[4] Wei G. F, Liu X. Y and Chen W. L (2009) "The Relativistic Scattering States of the Hulthen Potential with an Improved New Approximation Scheme to the Centrifugal Term" International Journal of Theoretical Physics, Vol. 48, No. 6, pp. 1649-1658. doi:10.1007/s10773-009-9937-9. 
[5] Sever. R, Tezcan. C, Yesiltas. O and Bucurget. M, (2008) "Exact Solution of Effective Mass Schrodinger Equation for the Hulthen Potential," International Journal of Theoretical Physics, Vol. 47, No. 9, 2008, pp. 2243-2248. doi:10.1007/s10773-008-9656-7

[6] Ikot. A.N, Antia, A.D, Akpabio, L.E and Obu. A. J(2011) "Analytic Solutions of Schrodinger Equation with Two-Dimensional Harmonic Potential in Cartesian and Polar Coordinates via Nikiforov-Uvarov Method," Journal of Vectorial Relativity, Vol. 6, No. 2, pp. 65-76.

[7] Al-Dossary, O. M (2007) "Morse Potential Eigen-Energies through the Asymptotic Iteration Method," International Journal of Quantum Chemistry, Vol. 107, pp. 2040-2046. doi:10.1002/qua.21335

[8] Oyewumi K.J , Akinpelu O. E and Agboola A.D (2008) "Exactly Complete Solutions of the Pseudoharmonic Potential in N-Dimensions," International Journal of Theoretical Physics, Vol. 47, No. 4, pp. 1039-1057. doi:10.1007/s10773-007-9532-x

[9] Oluwadare O. J. , Oyewumi K. J. , Akoshile C. O. , and Babalola O. A (2012) "Approximate analytical solutions of the relativistic equations with the Deng-Fan molecular potential including a Pekeris-type approximation to the (pseudo or) centrifugal term", Phys. Scr. Vol. 86, 035002.

[10] Nikiforov A.F., Uvarov V. B (1988) Special Functions of Mathematical Physics, Birkhauser, Bassel

[11]Meyur S (2011) "Algebraic Aspect for Two Solvable Potentials," Electronic Journal of Theoretical Physics, Vol. 8, No. 25, pp. 217-224.

[12] Hassanabadi H., Maghsoodi E., Zarrinkamar S., and Rahimov. H (2012) "Dirac equation under scalar, vector and tensor cornell interactions", Adv. High Energy Phys. 707041.

[13] Okon I.B., Popoola O and Isonguyo, C. N ( 2014), "Exact Bound state Solution of Q-deformed Woods-Saxon Plus modified Coulomb Potential Using Conventional Nikiforov-Uvarov Method", International Journal of Recent Advances In Physics, Vol.3, No.4, pp29-38.

[14] Hassanabadi H., Zarrinkamar S. and Rajabi A.A. (2011) "Exact solutions of D-dimensional schrödinger equation for an energy-dependent potential by NU method" Commun. Theor. Phys. Vol. 55 , pp 541

[15] Junker G (1996) "Supersymmetric methods in Quantum and statistical Physics" Springer-Verlag.

[16]Dong S.H., Factorization Method in Quantum Mechanics. (2007). Springer-Verlag, Berlin

[17]Wei . G. F and Dong S.H (2011) "Algebraic approach to energy spectra of the Scarf- Type and generalized Poschl-Teller potentials", Canadian Journal of Physics Vo. 89, pp1225-1231.

[18] Wei . G. F., Long, C.Y, Duan, XY and Dong S.H (2008) "Arbitrary l-wave scattering state solutions of the Schrodinger equation for the Eckart potential ", Physica Scripta Vo. 89, pp1225-1231

[19] Setare M. R and Hatami O, (2008), "Exact Solutions of the Dirac Equation for an electron in magnetic field with shape invariant method". Chinese Physics Letter, Volume 25, 035001.

[20]Landau L. D. and Lifshitz E. M. (1977). Quantum Mechanics, Non-Relativistic Theory, Pergamon, New York, NY, USA, 3rd edition.

[21] S. H. Dong (2001) Schrodinger Equation with the potential .Phys. Scr. 64 pp 273

[22] Hassanabadi H., Zarrinkamar S. and Rajabi A.A. (2011) "Exact solutions of D-dimensional schrödinger equation for an energy- dependent potential by NU method" Commun. Theor. Phys. Vol. 55, pp 541.

[23] Pekeris,C. L (1934) "The rotation-vibration coupling in diatomic molecules", Physical Review, vol. 45, No. 2, pp. 98-103.

[24] Sadeghi, j and Pourhassan, B.(2008) "The Exact Solution of the Non - Central Modified Kratzer Potential Plus a Ring - Shaped like potential by the factorization method", Electronic Journal of Theoretical Physics: Vol.17 pp $193-202$.

[25] Ikhdair, S. M and Sever, R (2008) "Exact solutions of the modified kratzer potential plus ring-shaped potential in the D-dimensional Schrodinger equation by the Nikiforov-Uvarov method", International Journal of Modern Physics C, vol. 19, No. 2, pp. 221-235.

[26] Berkdemir, C., Berkdemir, A and Han,J (2006) "Bound state solutions of the Schrodinger equation for modified Kratzer's molecular potential" Chemical Physics Letters, vol. 417, No. 4 pp. 326-329.

[27] Ikhdair S. M. and Sever R, ( 2007). "An alternative simple solution of the sextic anharmonic oscillator and perturbed coulomb problems," International Journal of Modern Physics C, vol. 18, No10, pp1571. 
International Journal of Recent advances in Physics (IJRAP) Vol.4, No.3, August 2015

[28] Alhaidari, A.D., Bahlouli, H.,Al-Hasan,A. (2006) "Dirac and Klein-Gordon equations with equal scalar and vector potentials. Phys. Lett A, vol 349 Lett. A 349, 87-97

[29] Berkdemir, C.(2006) " Pseudospin symmetry in the relativistic Morse potential including the spinorbit coupling term” Nucl. Phys.A 770, pp 32-39

[30] Dutt, R., Chowdhury, K., Varshni, Y.P(1985) An improved calculation for screened Coulomb potentials in Rayleigh-Schrodinger perturbation theory. J. Phys. A: Math. Gen. vol. 18, pp1379-1388. 